\title{
Household poverty, schooling, stigma and quality of life in adolescents with epilepsy in rural Uganda
}

\author{
Ronald Anguzu a,b,c , Pamela Akun a,b, Thomas Katairo a , Catherine Abbo a , Albert Ningwa ${ }^{\mathrm{a}, \mathrm{b}}$, \\ Rodney Ogwang ${ }^{\mathrm{a}, \mathrm{b}, \mathrm{d}}$, Amos Deogratius Mwaka ${ }^{\mathrm{a}}$, Kevin Marsh ${ }^{\mathrm{e}}$, Charles R. Newton ${ }^{\mathrm{d}, \mathrm{f}}$, Richard Idro ${ }^{\mathrm{a}, \mathrm{b}, \mathrm{d}, \mathrm{e}, *}$ \\ ${ }^{a}$ Makerere University College of Health Sciences, Kampala, Uganda \\ ${ }^{\mathrm{b}}$ Centre for Tropical Neuroscience, Kampala, Uganda \\ ${ }^{c}$ Institute of Health and Equity, Medical College of Wisconsin, Milwaukee, WI, USA \\ ${ }^{\mathrm{d}}$ Kenya Medical Research Institute-Wellcome Trust Collaborative Programme, Kilifi, Kenya \\ ${ }^{\mathrm{e}}$ Centre for Tropical Medicine and Global Health, University of Oxford, Oxford, UK \\ ${ }^{\mathrm{f}}$ Department of Psychiatry, University of Oxford, Oxford, UK
}

\section{A R T I C L E I N F O}

\section{Article history:}

Received 31 August 2020

Revised 20 October 2020

Accepted 21 October 2020

Available online 25 November 2020

\section{Keywords:}

Adolescent

Convulsive epilepsy

Poverty

Schooling

Stigma

Quality of Life

\begin{abstract}
A B S T R A C T
Background: Epilepsy remains a leading chronic neurological disorder in Low- and Middle-Income Countries. In Uganda, the highest burden is among young rural people. We aimed to; (i) describe socio-economic status (including schooling), and household poverty in adolescents living with epilepsy (ALE) compared to unaffected counterparts in the same communities and (ii) determine the factors associated with the overall quality of life (QoL).

Methods: This was a cross-sectional survey nested within a larger study of ALE compared to age-matched healthy community children in Uganda. Between Sept 2016 to Sept 2017, 154 ALE and 154 healthy community controls were consecutively recruited. Adolescents recruited were frequency and age-matched based on age categories 10-14 and 15-19 years. Clinical history and standardized assessments were conducted. One control participant had incomplete assessment and was excluded. The primary outcome was overall QoL and key variables assessed were schooling status and household poverty. Descriptive and multivariable linear regression analysis were conducted for independent associations with overall QoL.

Results: Mean (SD) age at seizure onset was 8.8 (3.9) years and median (IQR) monthly seizure burden was 2 (1-4). Epilepsy was associated with living in homes with high household poverty; 95/154 (61.7\%) ALE lived in the poorest homes compared to $68 / 153(44.5 \%)$ of the healthy adolescents, $p=0.001$. Nearly twothirds of ALE had dropped out of school and only 48/154 (31.2\%) were currently attending school compared to $136 / 153$ (88.9\%) of healthy controls, $p<0.001$. QoL was lowest among ALE who never attended school $(p<0.001)$, with primary education $(p=0.006)$ compared to those with at least secondary education. Stigma scores [mean(SD)] were highest among ALE in the poorest [69.1(34.6)], and wealthy [70.2 (32.2)] quintiles compared to their counterparts in poorer [61.8(31.7)], medium [68.0(32.7)] and wealthiest [61.5(33.3)] quintiles, though not statistically significant $(p=0.75)$. After adjusting for covariates, ALE currently attending school had higher overall QoL compared to their counterparts who never attended school $(\beta=4.20,95 \% \mathrm{CI}: 0.90,7.49, p=0.013)$. QoL scores were higher among ALE with $\geq$ secondary education than those with no or primary education ( $\beta=10.69,95 \% \mathrm{CI}: 1.65,19.72)$.

Conclusions: ALE in this rural area are from the poorest households, are more likely to drop out of school and have the lowest QoL. Those with poorer seizure control are most affected. ALE should be included among vulnerable population groups and in addition to schooling, strategies for seizure control and addressing the epilepsy treatment gap in affected homes should be specifically targeted in state poverty eradication programs.
\end{abstract}

(c) 2020 Published by Elsevier Inc.
* Corresponding author at: Makerere University College of Health Sciences, P.O Box 7072, Kampala, Uganda.

E-mail address: ridro1@gmail.com (R. Idro).

\section{Introduction}

Epilepsy is a leading chronic neurological disorder characterized by a propensity to recurrent unprovoked seizures [1-3]. This 
global health problem affects over 50 million people of all ages. Over $80 \%$ of people with epilepsy live in Low and Middle-Income Countries (LMICs) [4-6]. In these regions, populations in rural areas bear a greater burden of the disease: with an estimated median prevalence of active convulsive epilepsy of 12.7 compared to 5.9 per 1000 population in urban areas [7]. The annual mortality is high at a rate of 19.8 deaths (range 9.7-45.1) per 1000 people with epilepsy [8] with a standardized mortality ratio (SMR) of 7.2 (95\% CI 4.4-11.6) [9].

The burden of active convulsive epilepsy varies across subSaharan Africa and differences in the prevalence of risk factors contribute to the variation in burden [10]. Although the relative contributions of the specific causes of epilepsy have not been fully investigated, the most important include birth trauma, childhood central nervous system (CNS) infections such as bacterial meningitis and cerebral malaria [11-15], onchocerciasis [9,16,17], cysticercosis [18], and traumatic brain injury [19]. Many of these etiological factors are more common in poorer communities suggesting that in poor countries, poverty is a major risk factor of preventable epilepsy.

Epilepsy has devastating consequences on patient's wellbeing such as stigma, reduced likelihood of employment, as well as physical and psychological vulnerability [20]. In rural settings, large family sizes, poor financial returns from agriculture, low household incomes, parental unemployment, and stigma towards children with epilepsy (CLE) all affect families' capacity to address the basic needs of CLE. Moreover, the socio-economic status of homes, parental beliefs, education and household poverty have a negative impact on access to care, achieving seizure control and so, may be important drivers of the higher burden and severity of epilepsy in Africa and the increased attributable mortality [21,22]. Childhood poverty remains highest in the Northern regions of Uganda [23].

Adolescence is an important transitional stage of life between childhood to adulthood [24]. Many life events during adolescence have the potential for long-term impact including psychosocial, overall earnings and socio-economic status [25]. Some national advocacy efforts for poverty reduction have prioritized supporting livelihoods in youths below 35 years [26]. Most studies of epilepsy in Africa have also focused on young children and adults to examine the burden, features [27], risks factors [28], antiepileptic drug treatment [29] or clinical [19] and policy implications. To our best knowledge, there are almost no studies of epilepsy in adolescents or the impact of epilepsy on livelihood, achievement, and quality of life (QoL) in the region. Here, we describe schooling, socioeconomic status and household poverty and the relationship of these with QoL of adolescents living with epilepsy (ALE) in rural Uganda compared to unaffected adolescents in the same communities. We hypothesized that QoL scores in adolescents with epilepsy differed by their level of formal schooling and household poverty.

\section{Material and methods}

\subsection{Study design}

This was a cross sectional study describing the socio-economic status, household poverty, attendance of formal education, and QoL of adolescents with epilepsy compared to age-matched normal community children in northern Uganda. The study was nested in a large project studying the pathogenesis and treatment of a devastating neurologic disorder in this region, the nodding syndrome, and Onchocerca volvulus associated epilepsy [30]. Ethical approval was provided by Makerere University School of Medicine Research and Ethics Committee (SOMREC), REC Ref: 2016-022, University of
Oxford Tropical Medicine Research Ethics Committee (OXTREC), Ref: 12-16 and Uganda National Council for Science and Technology (UNCST), HS 1986.

\subsection{Study setting}

The study was carried out in the districts of Pader, Kitgum and Lamwo located in Northern region of Uganda. The population in the three districts is predominately Acholi, a Luo speaking tribe, and the main economic activity is subsistence agriculture on small farmlands. In 2006, these districts emerged from a two-decade trauma of the Lord's Resistance Army war against the Ugandan Government [31]. Psycho-social problems in this study setting are a confluence of direct and indirect effects of decades of war in the area that led to poverty and poorer household QoL. These psycho-social problems include psychological distress coupled by loss of life in families and communities [32], abject poverty, poor access to healthcare services [33] and childhood malnutrition specifically stunting [34]. Other common social problems include harmful cultural practices, stigma, negative stereotypes from healthcare workers [35] and caregivers [36] towards patients with mental health problems. The area is highly endemic for malaria [37] and Onchocerca volvulus [38]. The three districts are also the most affected by the devastating nodding syndrome, which is characterized by severe epilepsy and over $75 \%$ of all nodding syndrome patients in Uganda live here [39].

\subsection{Study participants}

Participants were a sub-sample of the ongoing case-control study examining the pathogenesis of nodding syndrome. Participants with epilepsy were adolescents' aged 10-19 years, with active convulsive epilepsy $[2,22,40]$ as defined by the International League against Epilepsy as two or more unprovoked seizures occurring at least 24 hours apart. Recruited study participants were attending epilepsy care services at the nodding syndrome treatment centres in any of the three districts. Comparative participants were age-matched, healthy community children, aged 1019 years, living in the same community and with no known identifiable seizure disorders or other known chronic medical disorders. Patients living with NS who were attending NS treatment centers in these three districts [41] were not recruited into the current study.

\subsection{Study procedures}

\subsubsection{Sample size estimation}

The two sets of participants were age-matched in a 1:1 frequency ratio and the sample size of 154 per group was estimated using Connett's un-conditional approach that assumed a power $=0.8, \alpha=0.05$, desired odds ratio of 3.0. Frequency matching was based on age categories $10-14$ and $15-19$ years.

\subsubsection{Participant enrolment}

Adolescents with epilepsy, ages $10-19$ years, and their parents or care givers attending epilepsy clinics in the three districts, were informed of the study at least a week prior to the specific enrollment date. On the appointment date, a study assistant reintroduced the study, its objectives, and the procedures in a general talk to all patients and parents. Individual written parental consent and assent (from the adolescents) was obtained before initiating the study procedures. At the end of the procedures, participants and parents were asked if there were normal or epilepsy unaffected children of similar ages in the neighboring homes. If so, these were invited to participant as control community children. Socio-cultural and economic status of participants' neighbor- 
hoods from which ALE and the age-matched healthy controls were selected are similar.

\subsubsection{Study assessments}

Participants had standardized assessments including a description of the demographic characteristics (age, gender, residence, distance from nearest health unit, anthropometry (weight [kg] and height $[\mathrm{m}]$ ) for nutritional status assessed against WHO 2000 anthropometric standards, the family's socio-economic status. Schooling status was reported as having dropped out of school, never or currently attended formal school. Detailed clinical history taking, full physical exam, epilepsy treatment and cognitive testing was conducted. The clinical history included a description of the past medical history, the progressive development of symptoms, and the types and burden of seizures. The burden of seizures was reported as the number in the past month. Socio-economic status or wealth index was used as a marker of household poverty and measured using a household asset index computed from household items and property owned by the family as well as parental earning as previously described [42].

Quality of life was assessed using the Quality of Life in Childhood Epilepsy (QoLCE) questionnaire. The QoLCE is a tool that assesses a child's physical and social activity, cognition, wellbeing, behaviour and general health. QoLCE was administered to parents of children with epilepsy and each QOLCE domain is measured by a series of items measured on a Likert 6-scale [43].

\subsubsection{Study outcome}

The study outcome measure was overall QoL. Other key variables were schooling and education level, and the socioeconomic status or wealth index.

\subsection{Data management and statistical analysis}

Epi info version 7.1.5.2 and Microsoft Access were used for data entry. Data were exported and analyzed in Stata v14.0 (STATA CORP, Texas). Continuous variables were summarized using means and their standard deviations and differences tested using the Stu- dent's t-test. Categorical variables were summarized as frequencies and proportions and the Chi-square test used to test for differences between children with epilepsy and the healthy adolescents. Fisher's exact test was used where cells had less than 5 observations.

Linear transformation of all QOLCE domain scores into scales of 0-100 points was done with higher scores representing better QoL. "Not applicable" responses to any question were coded as missing values. Cronbach's alpha was run to test for reliability of QOLCE subscales and observed to be 0.89 in our study population. For household poverty, principal component analysis was run converting the wealth index into a predicted wealth-score. From the wealth score, a wealth quintile was generated, defined as Poorest, Poor, Medium, Wealthy, and Wealthiest. To determine the relationship between household poverty, schooling status and education level with the different domains of QoL, one-way ANOVA was used and the differences between the different means and standard deviations for each level were compared. The omnibus p-value was reported and a $p$-value $<0.05$ was considered statistically significant.

To determine the factors associated with overall QoL among the adolescents with epilepsy, simple linear regression was used to determine crude coefficients $(\beta)$. Variables whose crude coefficients with a $p$-value $<0.2$ were included in the multivariable linear regression model namely schooling status, education level, seizure frequency and age of first seizure onset. In addition, variables that are known to be associated with QoL (age, sex, household wealth index) were also included in the final model. Before fitting the multiple linear regression, assumptions of linearity, independence, normality of the residuals and homoscedasticity were tested. Multi-collinearity and influential observations were also checked for before the model was fit. Using backward stepwise procedure with the probability of removal set at 0.05 , variables were omitted leaving statistically significant variables only. The dropped variables were tested for their confounding influence on the retained variables and a variable was considered a confounder if it changed the coefficients by over $10 \%$. Variables were considered statistically significant if they had a $p$-value $<0.05$ and they were presented with their corresponding 95\% confidence intervals.

Table 1

Characteristics of epileptic and healthy adolescents in rural Uganda, $N=307$.

\begin{tabular}{|c|c|c|c|}
\hline Characteristic & $\begin{array}{l}\text { Adolescents with epilepsy, } \\
N=154 \\
n(\%)\end{array}$ & $\begin{array}{l}\text { Healthy controls } \\
N=153 \\
n(\%)\end{array}$ & $p$-value \\
\hline Age $(\text { Mean } \pm S D)^{*}$ & $15.5 \pm 1.9$ & $14.7 \pm 1.9$ & $<0.001$ \\
\hline Sex, male & $93(60.4)$ & $84(54.9)$ & 0.331 \\
\hline Household poverty level & & & 0.001 \\
\hline Poorest & $38(24.7)$ & $20(13.1)$ & \\
\hline Poor & $57(37.0)$ & $48(31.3)$ & \\
\hline Medium & $25(16.2)$ & $22(14.4)$ & \\
\hline Wealthy & $21(13.6)$ & $26(17.0)$ & \\
\hline Wealthiest & $13(8.4)$ & $37(24.2)$ & \\
\hline Schooling & & & $<0.001$ \\
\hline Never attended school & $6(3.9)$ & $1(0.7)$ & \\
\hline Currently in school & $48(31.2)$ & $136(88.9)$ & \\
\hline Dropped out of school & $100(64.9)$ & $16(10.5)$ & \\
\hline Highest education level achieved* & & & 0.378 \\
\hline Primary school & $144(97.3)$ & $144(94.7)$ & \\
\hline Secondary school or higher & $4(2.7)$ & $8(5.3)$ & \\
\hline \multicolumn{4}{|l|}{ Childhood development } \\
\hline Had term birth & $134(87.0)$ & $145(94.8)$ & 0.018 \\
\hline Developmental concerns by 3 years & $7(4.5)$ & $4(2.6)$ & 0.363 \\
\hline Ever lived in IDP & $147(95.5)$ & $140(91.5)$ & 0.161 \\
\hline Other siblings with epilepsy & $54(35.1)$ & $39(25.7)$ & 0.074 \\
\hline Number of siblings with epilepsy & & & 0.049 \\
\hline 1 & $38(70.4)$ & $35(89.7)$ & \\
\hline$\geq 2$ & $16(29.6)$ & $4(10.3)$ & \\
\hline
\end{tabular}

\footnotetext{
${ }^{*}$ Years, IDP-Internally Displaced Peoples' camp.
} 


\section{Results}

\subsection{Demographics}

Over one year from September 2016 to September 2017, 154 patients with convulsive epilepsy and 154 age-matched healthy community controls were recruited. One community control had incomplete assessments and was excluded. The mean (SD) age of participants with epilepsy and healthy controls was 15.5 (1.9) and 14.7 (1.9) years respectively. Three fifths (60.4\%) of the ALE were male. The mean (SD) age at seizure onset was 8.8 (3.9) years and at the time of this study, the median (IQR) monthly seizure burden was $2(1-4)$ (Table 1$)$.

\subsection{Epilepsy in rural Uganda is associated with high levels of school dropout and poverty}

Epilepsy among adolescents was associated with living in households with high poverty levels. Overall, a total of 95/154 $(61.7 \%)$ of adolescents with epilepsy lived in the poorest households (the two lowest household poverty categories) compared to $68 / 153(44.5 \%)$ of the healthy adolescents, $p=0.001$. The proportion of adolescents with epilepsy living in the poorest households was twofold that of healthy controls $(24.7 \%$ vs $13.1 \%, p=0.001)$. Nearly $2 / 3$ of adolescents with epilepsy had dropped out of school. In addition, the proportion of adolescents with epilepsy who dropped out of school was six-fold that of healthy adolescents (64.9\% vs $10.5 \%, p<0.001$ ). At the time of the survey, only $31.2 \%$ of patients with epilepsy were currently attending school compared to $88.9 \%$ of healthy controls, $p<0.001$ (Table 1 ).

\subsection{Adolescents with epilepsy have a lower QoL compared to healthy controls}

The QoL domains among adolescents with epilepsy associated with schooling were physical $(p=0.002)$, attention $(p<0.001)$, memory $(p=0.002)$, language $(p<0.001)$, self-esteem $(p=0.003)$, interaction $(p=0.001)$, activities $(p=0.012)$, behavior $(p=0.002)$, general health $(p=0.012)$, general QoL $(p=0.004)$ and overall QoL ( $p<0.001$ ), (Table 2). Stigma scores were highest among adolescents in the poorest [mean $(\mathrm{SD})=69.1$ (34.6)], and wealthy [mean (SD) $=70.2(32.2)$ ] quintiles compared to their counterparts in poorer [mean $(S D)=61.8(31.7)$ ], medium [mean $(S D)=68.0$ (32.7)] and wealthiest [mean (SD) $=61.5$ (33.3)] quintiles. However, this association between stigma and household wealth was not statistically significant $(p=0.75)$.

After adjusting for covariates, ALE who are currently attending school had significantly higher overall QoL scores compared to their counterparts who never attended school $(\beta=4.197,95 \% \mathrm{CI}$ : 0.902, 7.493). Overall QoL scores were 10.69 higher among ALE who had secondary school education or higher than their counterparts who never attended or had primary level education ( $\beta=10.685,95 \% \mathrm{CI}: 1.653,19.717)$ as shown in Table 3 . In addition, each unit increase in monthly seizure frequency significantly reduced QoL scores by 0.66 ( $\beta=-0.66,95 \% \mathrm{CI}$ : $-0.95,-0.37)$ while QoL significantly reduced by 0.89 with each unit increase in age of onset of first seizure $(\beta=0.89,95 \% \mathrm{CI}$ : $0.48,1.30)$.

\section{Discussion}

This study aimed to examine schooling, household poverty and QoL of adolescents with epilepsy in rural northern Uganda. The study demonstrated that epilepsy in adolescents is associated with higher levels of household poverty and poorer QoL. To the best of our knowledge, this is the first such study to describe the relation-

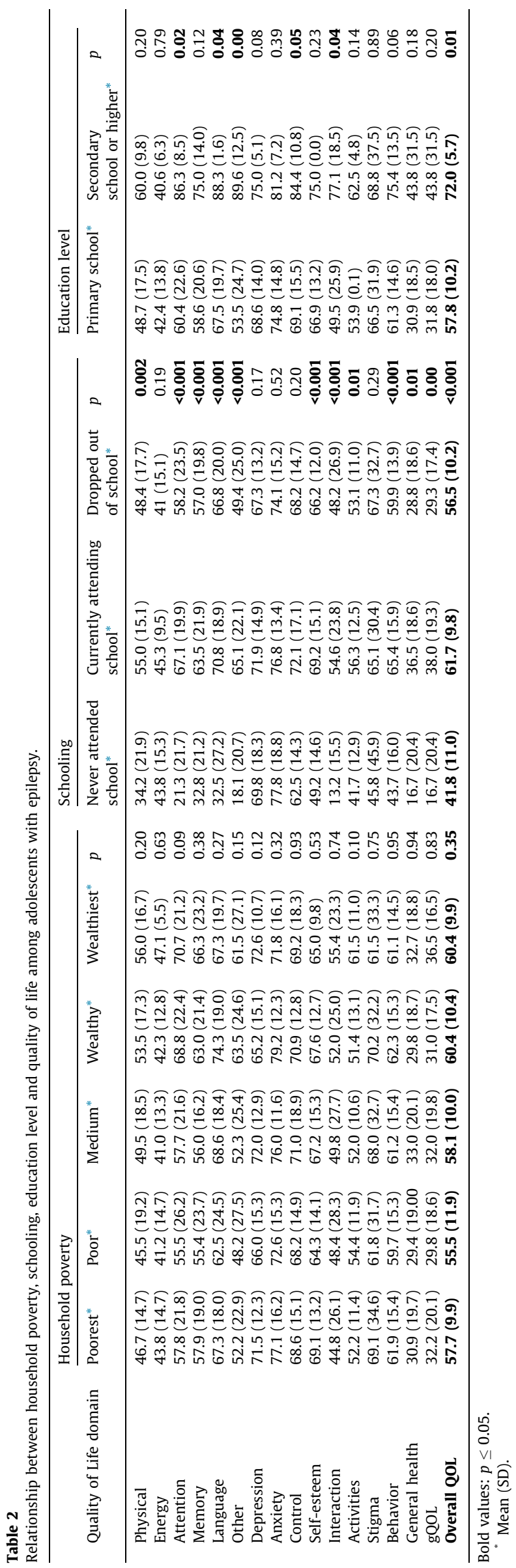


Table 3

Adjusted regression analysis for factors independently associated with quality of life among adolescents with epilepsy in rural Uganda.

\begin{tabular}{|c|c|c|c|c|c|}
\hline & \multicolumn{2}{|c|}{ Unadjusted } & \multicolumn{3}{|l|}{ Adjusted } \\
\hline & Crude $\beta$ & $p$ & Coef. & $(95 \% \mathrm{CI})$ & $p$ \\
\hline Age* & 0.18 & 0.712 & -0.62 & $(-1.54,0.31)$ & 0.189 \\
\hline \multicolumn{6}{|l|}{ Sex } \\
\hline Male & Ref & & Ref & & \\
\hline Female & -1.86 & 0.297 & -0.87 & $(-3.88,2.14)$ & 0.568 \\
\hline \multicolumn{6}{|l|}{ Schooling status } \\
\hline Never attended & Ref & & Ref & & \\
\hline Dropped out & 14.71 & 0.001 & Omitted & & \\
\hline Currently attends & 19.94 & $<0.001$ & 4.20 & $(0.90,7.49)$ & 0.013 \\
\hline \multicolumn{6}{|l|}{ Highest education level } \\
\hline Never attended or /Primary & Ref & & Ref & & \\
\hline Secondary or higher & 14.18 & 0.006 & 10.69 & $(1.65,19.72)$ & 0.021 \\
\hline \multicolumn{6}{|l|}{ Household wealth index } \\
\hline Poorest & Ref & & Ref & & \\
\hline Poor & -2.20 & 0.332 & -2.25 & $(-6.02,1.53)$ & 0.242 \\
\hline Medium & 0.37 & 0.895 & -3.48 & $(-8.08,1.13)$ & 0.138 \\
\hline Wealthy & 2.70 & 0.358 & 0.71 & $(-4.22,5.64)$ & 0.776 \\
\hline Wealthiest & 2.72 & 0.433 & -0.76 & $(-6.61,5.08)$ & 0.797 \\
\hline Monthly Seizure frequency & -0.75 & $<0.001$ & -0.66 & $(-0.95,-0.37)$ & $<0.001$ \\
\hline Age of First seizure age onset & 0.87 & $<0.001$ & 0.89 & $(0.48,1.30)$ & $<0.001$ \\
\hline
\end{tabular}

Bold values: $p \leq 0.05$.

* Years.

ship between household poverty, schooling status and QoL among adolescent with epilepsy in this region.

\subsection{Epilepsy and schooling status}

We found that adolescents with epilepsy were less likely to be currently attending school when compared to healthier adolescents. In agreement with our findings, nearly half of the children under fifteen years of age presenting with seizures in a health facility-based survey in rural Western Uganda were not attending school [27]. Similarly, another observational study among schoolgoing children in Kitgum showed that nodding syndrome, an epileptic disorder of childhood-onset in onchocerciasis endemic regions in Africa [44-47] was significantly associated with school dropout [48]. Further, individuals with epilepsy may have poorer academic achievement due to absenteeism or their inability to attend school. School non-attendance may be as a result of uncontrolled or breakthrough seizures triggered by missed or under dosing of antiepileptic drugs [49,50]. Additionally, the consequences of poorly controlled seizures such as physical injuries and body burns may also contribute to school absenteeism or drop out [51].

Interestingly in our study, stigma was not significantly associated with schooling, poverty, and education. This is possibly due to high communal acceptability for epilepsy in children and adolescents than in adults because febrile seizure are easily explainable as an underlying cause [52]. Stigma is also based on fear that children and adolescents with epilepsy seem different as their seizures may be unpredictable and the cultural beliefs that epilepsy is infectious. Stigma may reduce when it becomes clear that the children and adolescents with epilepsy are unlikely to be unpredictable. It is possible that ongoing government interventions in the study area such as mass drug ivermectin treatment, larviciding and vector control for Oncocerchiasis [53,54] and the Doxycycline drug trial for the treatment of nodding syndrome $[30,41]$, seizures were markedly reduced thus the finding of no association of stigma with schooling, poverty and education.

On the contrary, one prior study showed that negative secondary effects of epilepsy include stigmatization from schools or communities with being an epileptic patient [55]. Kirabira and colleagues conducted a facility-based study among adolescent patients being managed for epilepsy and found that perceived stigma was more likely to be reported by children who never attended school [56]. Within communities, negative attitudes, inadequate knowledge of the disease and its causes are pervasive which may contribute to the stigmatization of epileptic patients [57]. This is likely to lead to missed opportunities or denial of schooling and future employment.

The long-term negative effects of epilepsy on human capital are likely to be higher with childhood-onset of epilepsy. Poverty increases the risk of worsening health outcomes such as symptomatic epilepsy, school dropouts and QoL. Negative attitudes, and stigma influences decision making and access to care by persons living with epilepsy and other severe mental disorders [58]. Kiwanuka et al. in 2018 found that people with epilepsy held negative beliefs, such as attributing the cause of epilepsy to evil spirits [59]. Northern Uganda experienced several decades of war linked to ethnic divisions and political control resulting in distrust towards government. This affected the adoption of government relief efforts by local communities for example towards alleviation of suffering in families affected by nodding syndrome in Northern Uganda [60]. In addition, evidence differs traditional healers being preferred as their main source of care [59] while others preferring to seek care from health facilities [58].

\subsection{Poverty and QoL}

After controlling for potential confounders, we demonstrated that adolescents with epilepsy have a higher likelihood of increased household poverty. This finding is consistent with previous reports suggesting that mental disorders have an increased risk of pushing individuals and families into chronic poverty when compared to the general population [61]. Similarly, epilepsy has also been linked to physical disability which may lead to chronic poverty [62]. Moreover, the social burden of epilepsy outweighs the medical burden [63]. Caregiver burden of epilepsy extends mostly to family members in epilepsy affected households [64]. Caregivers or parents may suffer reduced income due to time away from their sources of income or income-generating activities. In the long term, the economic impact of household poverty on epilepsy will includes inability to afford treatment costs [65], basic household or living needs, the high patient- caregiver burden [64]. 
We observed that about one quarter of healthy controls had up to two siblings living with epilepsy in their household which suggests potentially higher impact on household poverty and schooling status. Therefore, in the long term, this could be due to higher financial demands for treatment-related costs and continuation of education among households with controls compared to those with patients with epilepsy. To address our objective, among our population of interest who were adolescents with epilepsy only, we controlled for potential confounders in the final model.

\subsection{Schooling status and QoL}

Our study also found that adolescents with epilepsy who dropped out of school had lower overall QoL than those who were currently attending school. This suggests that the use of antiepileptic drugs for treatment improves the general QoL in patients with epilepsy. Conversely, studies shows that poor adherence and side-effects of AEDs affects school attendance [66] and performance [67]. We found that ALE who never attended school had lower overall QoL compared to their counterparts who dropped out of school or were currently attending school. The lower QoL subscales included the physical, behavior, interaction, memory, language, self-esteem, activities, and general health QoL. Studies elsewhere show that school going children with idiopathic epilepsy are more likely to experience failure in school or academic underachievement [68]. Dropping out of school could be due to poorly controlled seizures which often leads to physical or intellectual disabilities, behavioral and emotional problems. Furthermore, injuries following seizures such as burns or fractures from falls contributes to absenteeism from school. Studies show that symptomatic treatment of epileptic disorders improves clinical symptoms and behavioral outcomes [69] as well as the general QoL [70] in epileptic patients. Prior research in a survey of hospitals showed that overall QoL was mostly explained by social and mental functioning among patients with epilepsy [71] and QoL increased in patients who had surgery for intractable temporal lobe epilepsy [72].

Another key observation from our study was QoL among ALE reduced with increasing monthly seizure frequency and age of onset of first seizure. Prior research concurs with our findings showing that seizure frequency [73] and age of seizure onset reduces QoL. This is because increasing seizure frequency affects neurodevelopment especially in developing brains of adolescents resulting in physical, socio-emotional, learning, and cognitive difficulties. In the long term, independent living, physical and cognitive disability are negative outcomes that affects QoL in ALE often due to epilepsy related injuries, deformities, or comorbidities [56].

Regarding perceived stigma, studies have shown perceived stigma to negatively impact QoL through community stigma among post-surgical patients living epilepsy [72] and harmful cultural perceptions about epilepsy [74,75] affecting the health service utilization and subsequent general wellbeing of patients living with epilepsy. Prior research in Ugandan populations shows that older children and adolescents who have never attended school report feeling more stigmatized [56] due to prolonged duration of AED treatment. In fact, prior reviews recommend focusing on interventions targeting stigma reduction in order to improve epilepsy treatment outcomes and QoL [76].

\section{Study limitations}

First, this baseline survey was cross-sectional in design therefore we were limited in inferring causal relationships between poverty, schooling and QoL in adolescent living with epilepsy. Secondly, some factors associated with regular school attendance, completion rates and QoL in adolescents such as type of and adherence to AED, injury severity (burns) were not controlled for. Despite these methodological gaps, to our knowledge we present the first evidence from Northern Uganda demonstrating that adolescents with epilepsy live in poor homes with a lower QoL. Further, we show that epilepsy is associated with higher levels of poverty and predisposes them to long term poverty as children drop out of school.

\section{Implications for clinical practice and policy}

Quality of life is a multi-dimensional measure of individual general wellbeing therefore clinicians should consider assessing and interpreting associations between clinical history and symptoms within the patients' social context. We demonstrated the relationship between epilepsy and household poverty. A plausible explanation that links long term poverty levels in this setting due to high school dropouts particularly among adolescents with epilepsy can be drawn. Adolescents living with epilepsy should be included among vulnerable population groups and affected homes specifically targeted in state poverty eradication programs and interventions focused on schooling, seizure control and addressing the epilepsy treatment gap. In Uganda, such poverty eradication strategies include the Poverty Eradication Action Plan [77] and Health Sector Development Plan [78] whose strategic objectives are underscored for modification.

\section{Conclusions}

Adolescents living with Epilepsy in this rural area are from the poorest households, are more likely to drop out of school and have the lowest QoL. Those with poorer seizure control are most affected. ALE should be included among vulnerable population groups and in addition to schooling, strategies for seizure control and addressing the epilepsy treatment gap, affected homes should be specifically targeted in state poverty eradication programs.

\section{Declaration of Competing Interest}

All authors report no conflict of interest.

\section{Acknowledgements}

Thanks go to all respondents and their caregivers for their voluntary study participation. Gratitude goes to Center for Tropical Neurosciences team; Nelson Odoch, Innocent JJ Oryem, Deborah Akol, Alfred Aponi, Alfred Odong, Kisansa Mugoya, Stephen Okiror, and Kitgum General Hospital staff for their contributions to this project.

\section{Funding}

This study was funded by the Medical Research Council UK through a grant from the MRC/DFID African Research Leader scheme grant number MR/M025489/1 to Prof Kevin Marsh and Dr Richard Idro. The MRC and DFID had no role in the design of this study, during the execution, analysis, interpretation of the data, or decision to submit the results for publication.

\section{References}

[1] Fisher RS, Cross JH, D'souza C, French JA, Haut SR, Higurashi N, et al. Instruction manual for the ILAE 2017 operational classification of seizure types. Epilepsia 2017;58(4):531-42. 
[2] Scheffer IE, Berkovic S, Capovilla G, Connolly MB, French J, Guilhoto L, et al. ILAE classification of the epilepsies: position paper of the ILAE Commission for Classification and Terminology. Zeitschrift Fur Epileptologie 2018.

[3] Fisher RS. Final comments on the process: ILAE definition of epilepsy. Epilepsia 2014:55(4):492-3.

[4] WHO. Epilepsy: World Health Organization; 2019 [updated 20 June 2019. Available from: https://www.who.int/news-room/fact-sheets/detail/epilepsy.

[5] Moog JC, Kakooza-Mwesige A, Tan CT. Epilepsy in the tropics: emerging etiologies. Seizure 2017;44:108-12.

[6] Idro R, Newton C, Kiguli S, Kakooza-Mwesige A. Child neurology practice and neurological disorders in East Africa. J Child Neurol 2010;25(4):518-24.

[7] Ngugi AK, Bottomley C, Kleinschmidt I, Sander JW, Newton CR. Estimation of the burden of active and life-time epilepsy: a meta-analytic approach. Epilepsia 2010;51(5):883-90.

[8] Levira F, Thurman D, Sander J, Hauser W, Hesdorffer D, Masanja H, et al. Epidemiology Commission of the International League Against Epilepsy. Premature mortality of epilepsy in low-and middle-income countries: a systematic review from the Mortality Task Force of the International League Against Epilepsy. Epilepsia 2017;58(01):6-16.

[9] Kaiser C, Asaba G, Kasoro S, Rubaale T, Kabagambe G, Mbabazi M. Mortality from epilepsy in an onchocerciasis-endemic area in West Uganda. Trans R Soc Trop Med Hyg 2007;101(1):48-55.

[10] Ngugi AK, Bottomley C, Kleinschmidt I, Wagner RG, Kakooza-Mwesige A, AeNgibise K, et al. Prevalence of active convulsive epilepsy in sub-Saharan Africa and associated risk factors: cross-sectional and case-control studies. Lancet Neurol 2013:12(3):253-63.

[11] Evjen-Olsen B, Hinderaker SG, Lie RT, Bergsjø P, Gasheka P, Kvåle G. Risk factors for maternal death in the highlands of rural northern Tanzania: a casecontrol study. BMC Public Health 2008;8(1):52.

[12] Carter JA, Neville BG, White S, Ross AJ, Otieno G, Mturi N, et al. Increased prevalence of epilepsy associated with severe falciparum malaria in children. Epilepsia 2004;45(8):978-81.

[13] Christensen SS, Eslick GD. Cerebral malaria as a risk factor for the development of epilepsy and other long-term neurological conditions: a meta-analysis. Trans R Soc Trop Med Hyg 2015;109(4):233-8.

[14] Idro R, Karamagi C, Tumwine J. Immediate outcome and prognostic factors for cerebral malaria among children admitted to Mulago Hospital, Uganda. Ann Trop Paediatrics 2004;24(1):17-24.

[15] Idro R, Kakooza-Mwesige A, Balyejjussa S, Mirembe G, Mugasha C, Tugumisirize J, et al. Severe neurological sequelae and behaviour problems after cerebral malaria in Ugandan children. BMC Res Notes 2010;3(1):104.

[16] Kaiser C, Asaba G, Leichsenring M, Kabagambe G. High incidence of epilepsy related to onchocerciasis in West Uganda. Epilepsy Res 1998;30(3):247-51.

[17] Pion SD, Kaiser C, Boutros-Toni F, Cournil A, Taylor MM, Meredith SE, et al. Epilepsy in onchocerciasis endemic areas: systematic review and metaanalysis of population-based surveys. PLoS Negl Trop Dis 2009;3(6).

[18] Preux P-M, Druet-Cabanac M. Epidemiology and aetiology of epilepsy in subSaharan Africa. Lancet Neurol 2005;4(1):21-31.

[19] Ba-Diop A, Marin B, Druet-Cabanac M, Ngoungou EB, Newton CR, Preux P-M. Epidemiology, causes, and treatment of epilepsy in sub-Saharan Africa. Lancet Neurol 2014;13(10):1029-44.

[20] Baskind R, Birbeck GL. Epilepsy-associated stigma in sub-Saharan Africa: the social landscape of a disease. Epilepsy Behav 2005;7(1):68-73.

[21] Kariuki SM, Kakooza-Mwesige A, Wagner RG, Chengo E, White S, Kamuyu G, et al. Prevalence and factors associated with convulsive status epilepticus in Africans with epilepsy. Neurology 2015;84(18):1838-45.

[22] Newton CR, Garcia HH. Epilepsy in poor regions of the world. Lancet 2012;380 (9848):1193-201.

[23] Batana YM, Cockburn J, Kasirye I, Tiberti L, Ahaibwe G. Situation analysis of child poverty and deprivation in Uganda. Partnership for Economic Policy Working Paper. 2014(2014-03).

[24] Keeley B, Little C. The State of the Worlds Children 2017: Children in a Digital World: ERIC; 2017.

[25] Sheehan P, Sweeny K, Rasmussen B, Wils A, Friedman HS, Mahon J, et al. Building the foundations for sustainable development: a case for global investment in the capabilities of adolescents. Lancet 2017;390 (10104):1792-806.

[26] UNFPA Young People: The One Investment the Country Cannot Afford to Ignore 2017.

[27] Duggan M. Epilepsy and its effects on children and families in rural Uganda. African Health Sci 2013;13(3):613-23.

[28] Duggan M. Epilepsy in rural Ugandan children: seizure pattern, age of onset and associated findings. African Health Sci 2010;10(3).

[29] Atugonza R, Kakooza-Mwesige A, Lhatoo S, Kaddumukasa M, Mugenyi L, Sajatovic M, et al. Multiple anti-epileptic drug use in children with epilepsy in Mulago hospital, Uganda: a cross sectional study. BMC Pediatrics 2016;16 (1):34.

[30] Idro R, Anguzu R, Ogwang R, Akun P, Abbo C, Mwaka AD, et al. Doxycycline for the treatment of nodding syndrome (DONS); the study protocol of a phase II randomised controlled trial. BMC Neurol 2019;19(1):35.

[31] Pham PN, Vinck P, Stover E. Returning home: forced conscription, reintegration, and mental health status of former abductees of the Lord's Resistance Army in northern Uganda. BMC Psychiatry 2009;9(1):23.

[32] Buchmann K. 'You sit in fear': understanding perceptions of nodding syndrome in post-conflict northern Uganda. Global Health Action 2014;7(1):25069.
[33] Latio LSY, Nam NH, Shah J, Smith C, Sakai K, Stonewall Shaban K, et al. Economic burden of the persistent morbidity of nodding syndrome on caregivers in affected households in Northern Uganda. PLoS ONE 2020;15 (9):e0238643.

[34] Musisi S, Akena D, Nakimuli-Mpungu E, Abbo C, Okello J. Neuropsychiatric perspectives on nodding syndrome in northern Uganda: a case series study and a review of the literature. African Health Sciences 2013;13(2):205-18.

[35] Mutamba B, Abbo C, Muron J, Idro R, Mwaka A. Stereotypes on Nodding syndrome: responses of health workers in the affected region of northern Uganda. African Health Sciences 2013;13(4):986-91.

[36] Nakigudde J, Mutamba BB, Bazeyo W, Musisi S, James O. An exploration of caregiver burden for children with nodding syndrome (lucluc) in Northern Uganda. BMC Psychiatry 2016;16(1):255.

[37] Okello PE, Van Bortel W, Byaruhanga AM, Correwyn A, Roelants P, Talisuna A, et al. Variation in malaria transmission intensity in seven sites throughout Uganda. Am J Trop Med Hygiene 2006;75(2):219-25.

[38] Oguttu D, Byamukama E, Katholi CR, Habomugisha P, Nahabwe C, Ngabirano $\mathrm{M}$, et al. Serosurveillance to monitor onchocerciasis elimination: the Ugandan experience. Am J Trop Med Hygiene 2014;90(2):339-45.

[39] Idro R, Ogwang R, Kayongo E, Gumisiriza N, Lanyero A, Kakooza-Mwesige A, et al. The natural history of nodding syndrome. Epileptic Disord 2018;20 (6):508-16.

[40] Fisher RS. The new classification of seizures by the International League Against Epilepsy 2017. Curr Neurol Neurosci Rep 2017;17(6):48.

[41] Anguzu R, Akun PR, Ogwang R, Shour AR, Sekibira R, Ningwa A, et al. Setting up a clinical trial for a novel disease: a case study of the Doxycycline for the Treatment of Nodding Syndrome Trial-challenges, enablers and lessons learned. Global Health Action 2018;11(1):1431362.

[42] Chakraborty NM, Fry K, Behl R, Longfield K. Simplified asset indices to measure wealth and equity in health programs: a reliability and validity analysis using survey data from 16 countries. Global Health: Sci Practice 2016;4(1):141-54.

[43] Puka K, Goodwin SW, Ferro MA, Smith ML, Widjaja E, Anderson KK, et al. Validation of the Quality of Life in Childhood Epilepsy Questionnaire (QOLCE55 and QOLCE-16) for use by parents of young adults with childhood-onset epilepsy. Epilepsy Behav 2020;104:106904.

[44] WHO. Onchocerciasis 2020 [cited 2020 April 23]. Available from: https:// www.who.int/onchocerciasis/symptoms/nodding_syndrome/en/.

[45] Mwaka AD, Semakula JR, Abbo C, Idro R. Nodding syndrome: recent insights into etiology, pathophysiology, and treatment. Res Rep Trop Med 2018;9:89.

[46] CDC. Nodding Syndrome Centers for Disease Control and Prevention2012 [updated April 3, 2012. Available from: https://www.cdc.gov/globalhealth/ noddingsyndrome/default.htm.

[47] Hotterbeekx A, Lammens M, Idro R, Akun PR, Lukande R, Akena G, et al. Neuroinflammation and not tauopathy is a predominant pathological signature of nodding syndrome. J Neuropathol Exp Neurol 2019;78 (11):1049-58.

[48] Pacoto S. Factors associated with school dropout of pupils in Kitgum district: a case of Akwang sub county 2019.

[49] Frucht MM, Quigg M, Schwaner C, Fountain NB. Distribution of seizure precipitants among epilepsy syndromes. Epilepsia 2000;41(12):1534-9.

[50] Kaddumukasa M, Kaddumukasa M, Matovu S, Katabira E. The frequency and precipitating factors for breakthrough seizures among patients with epilepsy in Uganda. BMC Neurol 2013;13(1):182.

[51] Kariuki SM, Matuja W, Akpalu A, Kakooza-Mwesige A, Chabi M, Wagner RG, et al. Clinical features, proximate causes, and consequences of active convulsive epilepsy in Africa. Epilepsia 2014;55(1):76-85.

[52] O'Neill S, Irani J, Fodjo JNS, Nono D, Abbo C, Sato Y, et al. Stigma and epilepsy in onchocerciasis-endemic regions in Africa: a review and recommendations from the onchocerciasis-associated epilepsy working group. Infect Dis Poverty 2019;8(1):1-11.

[53] Gumisiriza N, Mubiru F, Fodjo JNS, Kayitale MM, Hotterbeekx A, Idro R, et al. Prevalence and incidence of nodding syndrome and other forms of epilepsy in onchocerciasis-endemic areas in northern Uganda after the implementation of onchocerciasis control measures. Infect Dis Poverty 2020;9(1):1-11.

[54] MoH. National Onchocerciasis Control Programme (NOCP). In: Division VC, editor. Ministry of health 2020.

[55] Ssebunnya J, Kigozi F, Lund C, Kizza D, Okello E. Stakeholder perceptions of mental health stigma and poverty in Uganda. BMC Int Health Human Rights 2009;9(1):5

[56] Kirabira J, Nakawuki M, Fallen R, Rukundo GZ. Perceived stigma and associated factors among children and adolescents with epilepsy in south western Uganda: a cross sectional study. Seizure 2018;57:50-5.

[57] Kaddumukasa M, Kakooza A, Kayima J, Kaddumukasa MN, Ddumba E, Mugenyi L, et al. Community knowledge of and attitudes toward epilepsy in rural and urban Mukono district, Uganda: a cross-sectional study. Epilepsy Behav 2016;54:7-11.

[58] Kisa R, Baingana F, Kajungu R, Mangen PO, Angdembe M, Gwaikolo W, et al. Pathways and access to mental health care services by persons living with severe mental disorders and epilepsy in Uganda, Liberia and Nepal: a qualitative study. BMC Psychiatry 2016;16(1):305.

[59] Kiwanuka F, Anyango Olyet C. Knowledge, attitude, and beliefs on epilepsy among adults in Erute south, Lira District, Uganda. Epilepsia Open 2018;3 (2):264-9.

[60] Irani J, Rujumba J, Mwaka AD, Arach J, Lanyuru D, Idro R, et al. “Those who died are the ones that are cured". Walking the political tightrope of Nodding 
Syndrome in northern Uganda: emerging challenges for research and policy. PLoS Negl Trop Dis 2019;13(6).

[61] Funk M, Drew N, Knapp M. Mental health, poverty and development. J Public Mental Health 2012.

[62] Lwanga-Ntale C. Chronic poverty and disability in Uganda. 2003.

[63] Birbeck GL, Chomba E, Atadzhanov M, Mbewe E, Haworth A. Women's experiences living with epilepsy in Zambia. Am J Trop Med Hygiene 2008;79 (2):168-72.

[64] Kaddumukasa M, Nalubwama H, Kaddumukasa MN, Lhatoo S, Sewankambo N, Katabira E, et al. Barriers to epilepsy care in Central Uganda, a qualitative interview and focus group study involving PLWE and their caregivers. BMC Neurol 2019:19(1):161.

[65] Allers K, Essue BM, Hackett ML, Muhunthan J, Anderson CS, Pickles K, et al. The economic impact of epilepsy: a systematic review. BMC Neurol 2015;15(1):245.

[66] Reilly C, Atkinson P, Das KB, Chin RF, Aylett SE, Burch V, et al. Academic achievement in school-aged children with active epilepsy: a population-based study. Epilepsia 2014;55(12):1910-7.

[67] Ibekwe R, Ojinnaka N, Iloeje S. Factors influencing the academic performance of school children with epilepsy. J Trop Pediatr 2007:53(5):338-43.

[68] De Alwis A, Kariyawasam S, Weerasinghe C. School drop out and fallout from idiopathic epilepsy in rural Sri Lanka. Sri Lanka J Child Health 2008;37(1).

[69] Idro R, Namusoke H, Abbo C, Mutamba BB, Kakooza-Mwesige A, Opoka RO, et al. Patients with nodding syndrome in Uganda improve with symptomatic treatment: a cross-sectional study. BMJ Open 2014;4(11):e006476.

[70] Korevaar DA, Visser BJ. Reviewing the evidence on nodding syndrome, a mysterious tropical disorder. Int J Infect Dis 2013;17(3):e149-52.
[71] Nabukenya AM, Matovu JK, Wabwire-Mangen F, Wanyenze RK, Makumbi F. Health-related quality of life in epilepsy patients receiving anti-epileptic drugs at National Referral Hospitals in Uganda: a cross-sectional study. Health Quality Life Outcomes 2014;12(1):49.

[72] Fletcher A, Sims-Williams H, Wabulya A, Boling W. Stigma and quality of life at long-term follow-up after surgery for epilepsy in Uganda. Epilepsy Behav 2015;52:128-31.

[73] Kaddumukasa M, Mugenyi L, Lhatoo S, Sewankambo N, Blixen C, Sajatovic M, et al. Seizure severity is associated with poor quality of life in people living with epilepsy (PLWE) in Uganda: a cross-sectional study. Epilepsy Behav 2019;96:104-8.

[74] Keikelame MJ, Suliaman T, Hendriksz M, Swartz L. Psychosocial challenges affecting the quality of life in adults with epilepsy and their carers in Africa: a review of published evidence between 1994 and 2014. Afr J Primary Health Care Family Med 2017;9(1):1-5.

[75] Boling W, Means M, Fletcher A. Quality of life and stigma in epilepsy perspectives from selected regions of Asia and Sub-Saharan Africa. Brain Sci 2018;8(4):59.

[76] Chakraborty P, Sanchez NA, Kaddumukasa M, Kajumba M, Kakooza-Mwesige A, Van Noord M, et al. Stigma reduction interventions for epilepsy: a systematized literature review. Epilepsy Behav 2020:107381.

[77] Fan S, Zhang X. Poverty reduction strategy paper: Uganda's poverty eradication action plan, summary and main objectives. Afr Develop RevRevue Africaine De Developpement 2008;20(3):466-96.

[78] MoH. Health sector and Development Plan 2015/16 - 2019/20. 2015. 